\title{
Rationale and Design of the Kento Heart Safe City Project:
}

\section{A First-aid System Using an Emergency Alarm Button (SOS Button)}

\author{
Yoshio Tahara ${ }^{1}$, Teruo Noguchi ${ }^{1}$, Kunihiro Nishimura ${ }^{2}$, Kenji Narikawa ${ }^{3}$, Hisao Ogawa ${ }^{1,4}$, and Satoshi Yasuda ${ }^{1,5}$
}

\begin{abstract}
Background: Although approximately 120,000 out-of-hospital cardiac arrests (OHCAs) occur annually in Japan, the rate of favorable neurological outcomes after cardiogenic cardiac arrests witnessed by the public is only $7-8 \%$. There are approximately 600,000 automated external defibrillators (AEDs) deployed nationwide, but the rate of AED use in patients with a publicly witnessed cardiac arrest is as low as $4.9 \%$. Methods: The Kento Heart Safe City Project is designed to increase the rate of AED use, reduce sudden cardiac deaths, and improve the rate of favorable neurological outcomes by using a newly developed emergency call button (SOS button by Philips Japan, Ltd.). This project includes training and properly deploying community first responders and developing an education and rapid notification system in an integrated manner. The SOS button is an Internet of Things (IOT) device and uses the same radio waves as cell phones; pressing the SOS button triggers the communication system. Discussion: Compared to ordinary emergency systems, the SOS button is a unique rescue system based on the premise of voluntary mutual aid. It is necessary to verify how many first responders will respond to SOS requests and how long it will take for responders to arrive at the scene.
\end{abstract}

KEY WORDS: automated external defibrillator, cardiac arrest, Internet of Things, resuscitation

\section{Introduction}

Approximately 120,000 out-of-hospital cardiac arrests (OHCAs) occur annually in Japan. Of these, the annual number of cardiogenic sudden arrests (sudden cardiac deaths) witnessed by the general public is about 30,000 , and approximately 80 people witness a sudden cardiac arrest somewhere in Japan every day. As cardiopulmonary resuscitation has become more widespread, the number of individuals with sudden cardiac arrest who return to society has doubled over the past 10 years. However, the overall rate at which they return to society remains low, at $8 \%{ }^{1)}$.

A 2002 survey in the Kanto region reported that $63 \%$ of witnessed out-of-hospital cardiac arrests were due to ventricular fibrillation $(\mathrm{VF})^{2}$. Electrical defibrillation is an effective treat-

${ }^{1}$ Department of Cardiovascular Medicine, National Cerebral and Cardiovascular Center, 6-1 Kishibe-shimmachi, Suita, Osaka 564-8565, Japan, ${ }^{2}$ Department of Preventive Medicine and Epidemiology, National Cerebral and Cardiovascular Center, ${ }^{3}$ Business Marketing Specialist, Connected Care, Emergency Care, Philips Japan, Ltd., ${ }^{4}$ Kumamoto University, ${ }^{5}$ Department of Cardiovascular Medicine, Tohoku University Graduate School of Medicine

E-mail: tahara@ncvc.go.jp

Received: February 15, 2021; Accepted: August 25, 2021

doi: 10.7793/jcad.27.21-00004

(C) The Japanese Coronary Association ment for VF. The widespread use of automated external defibrillators (AEDs) is expected to dramatically improve the rate at which individuals return to society after out-of-hospital cardiac arrest. In Japan, the use of AEDs by the general public was first permitted in 2004. Subsequently, the use of AEDs in Japan has become more widespread than in any other country in the world, with approximately 600,000 AEDs deployed nationwide. As the number of AEDs for general public use has increased, there has been an increase in the number of individuals who survive out-of-hospital cardiac arrests due to the use of AEDs by citizens $^{3)}$. However, since AEDs are used in only $5 \%$ of sudden cardiac arrests, there remains room for improvement ${ }^{1)}$.

For patients with VF in initial rhythm for which AEDs are effective, the rate of return of spontaneous circulation (ROSC) is $46 \%$, which is twice the rate of ROSC after electrical defibrillation by paramedics once they arrive at the scene $(20 \%)$. This result is related to the national average of 8.7 minutes from the time of the 119 emergency call to the time when the emergency team arrives at the scene. The ROSC rate of patients with VF depends on the time to electrical defibrillation. In the case of out-of-hospital cardiac arrest, electrical defibrillation within 5 minutes is required $^{1)}$. For this reason, the role of citizen rescuers at the scene is important. Recently, the International Liaison Committee On Resuscitation (ILCOR) International Consensus 2020 strongly recommended notifying dispatching- citizen near the scene of a suspected out-of-hospital cardiac arrest event and 
activating them through smartphone ${ }^{4)}$. A pilot study of a community-based volunteer network has been reported in the United States $^{5)}$. An alert is sent to a volunteer via text message when an emergency call is made. A study of the time from the emergency call to the arrival at the simulated residence showed that the volunteers were 4-6 minutes faster than the emergency medical services (EMS) team from the emergency call to the arrival of the AED.

Using Utstein-style emergency resuscitation statistics from the Fire and Disaster Management Agency of the Ministry of Internal Affairs and Communications in Japan, a study compared the ROSC rates of patients who had sustained VF when the EMS team arrived at the scene and those who underwent cardiopulmonary resuscitation (CPR) with or without an AED by the time when the EMS team arrived. The ROSC rate among patients who were resuscitated with AEDs was $38 \%$, which was significantly better than the $23 \%$ ROSC rate among those who were resuscitated without AEDs ${ }^{6}$. These results suggest that citizens who used AEDs might have prior knowledge about resuscitation, for instance as a result of attending a training course, and that the audio guidance provided by AEDs may have improved the quality of chest compressions, which are important in CPR. Therefore, community ties, regional activation, CPR education systems, emergency request systems, and other community medical initiatives are issues to be addressed ${ }^{7)}$.

\section{Kento Heart Safe City project}

Kento is an acronym for Knowledge, Exercise, Nutrition, and Town, all of which are necessary for the prevention of cardiovascular disease. Kento was built on the site of the former Suita Railroad Yard in the cities of Suita and Settsu in Osaka Prefecture. The National Cerebral and Cardiovascular Center moved to a new hospital in Kento on July 1, 2019. A variety of facilities are located in the Kento area, including the National Cerebral and Cardiovascular Center, Suita Municipal Hospital, and a commercial complex (Viera Kishibe Kento). As a new initiative in Kento, we have started a novel project called the Kento Heart Safe City project, which provides a rapid initial response to people who have collapsed, such as those who have suffered cardiopulmonary arrest (sudden cardiac death), through (1) proper placement of AEDs, (2) creation of a communication system (SOS button and My SOS application by Philips Japan, Ltd.), and (3) training of first responders who can properly operate AEDs (Fig. 1).

We will describe each topic of the Kento Heart Safe City project in the following sections. This study was conducted in accordance with the tenets of the Declaration of Helsinki. The study was approved by the Ethics Committee of National Cerebral and Cardiovascular Center (Ethical review approval number:
R19077).

\section{SOS button}

The Internet of Things (IoT) refers to objects that are connected to the Internet and that exchange data with other devices and systems without human input. Such objects include driverless automobiles and smart home appliances. The SOS button is an IoT device that connects a button to an application via the Internet (Fig. 2). It measures $3.3 \mathrm{~cm}$ (length) $\times 9.7 \mathrm{~cm}$ (width) $\times 1.4$ $\mathrm{cm}$ (thickness), weighs approximately $50 \mathrm{~g}$ (including batteries), and can be easily installed without any wiring using the LTE-M wireless communication standard. It can be linked with the free My SOS smartphone application to simultaneously notify registered users at the push of a button. In contrast to devices that use the Global Positioning System (GPS), the SOS button can display detailed information regarding the location of SOS activation, such as floor number and room number, if the location information has been registered in advance (Fig. 3). This function is applicable to individuals with COVID-19, for instance, who need to stay in their hotel room or in their home.

\section{Locations of AEDs and SOS buttons \& education}

Five AEDs were installed so that they could be reached within 5 minutes from anywhere in the demonstration area in Kento (Fig. 4), which is approximately $2 \mathrm{~km}$ in the east-west direction and $1 \mathrm{~km}$ in the north-south direction. In addition, SOS buttons were installed at intervals of 200 meters. First responders are persons who voluntarily rush to the scene after pressing the SOS button or receiving an SOS request from a smartphone terminal. They are employees of nearby facilities and members of the public in the Kento area who have taken cardiopulmonary resuscitation training beforehand and understand the SOS button system and are willing to participate in this project. Based on the number of passengers who get on and off at the JR Kishibe Station $(15,591$ passengers/day) and the number of first responders in the vicinity of the station, approximately 10 SOS button activations are expected each year. In addition, based on the total number of employees at schools $(n=25)$, hospitals $(n=45)$, and commercial facilities $(n=58)$ in the area, training sessions were held at the National Cerebral and Cardiovascular Center in July 2019 and at Osaka Gakuin University in January 2020, with the goal of training up to 1,000 first responders. In order to verify whether the first responder was able to respond to SOS button activation and the time required for the first responder to arrive at the scene, we first set up a demonstration period from March 2, 2020 to February 28, 2021 and then officially started the study.

\section{Different methods of notifying first responders}

When a witness of an out-of-hospital cardiac arrest calls for 


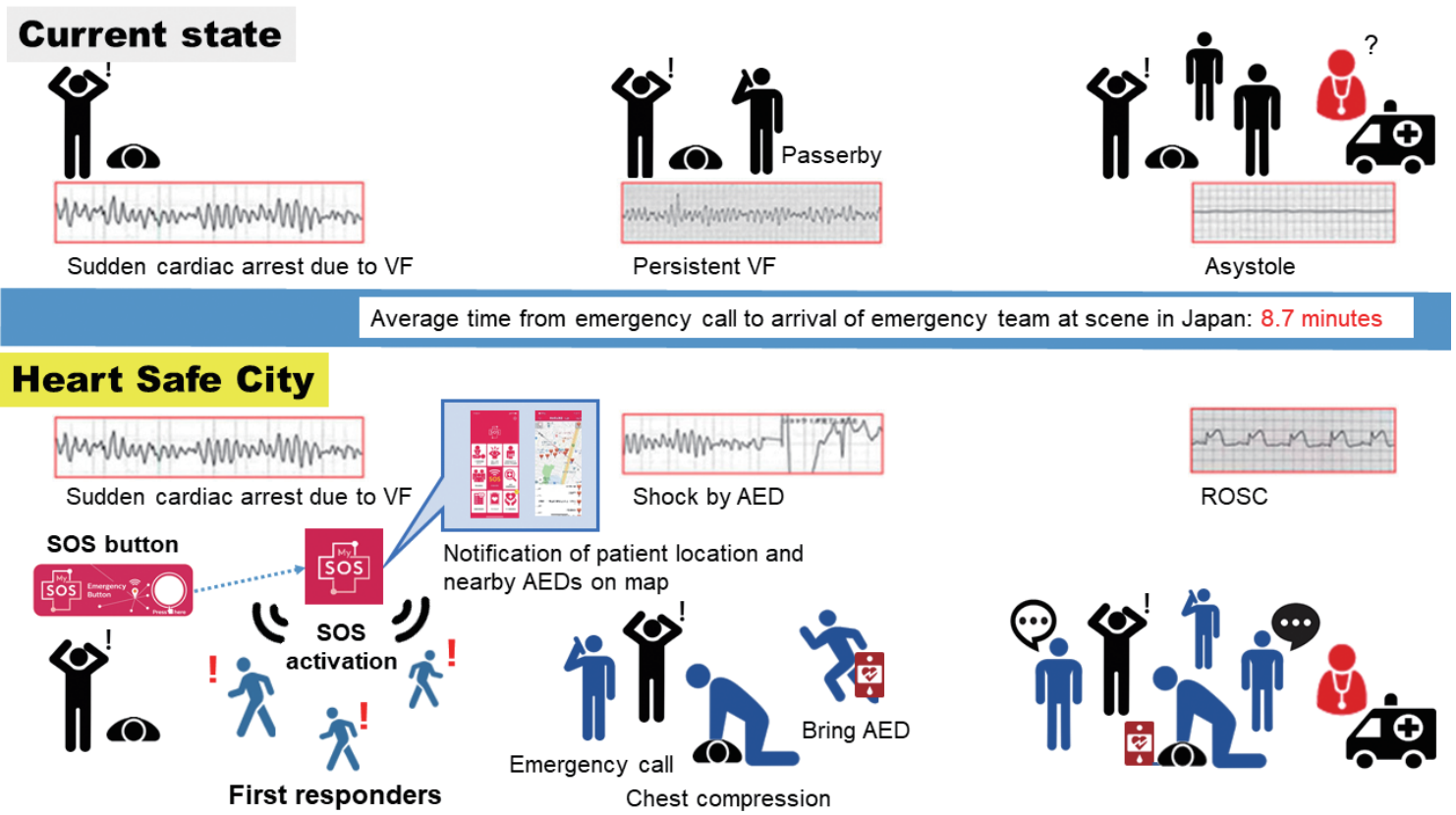

Fig. 1 Kento Heart Safe City project.

Kento Heart Safe City is a citywide effort to create a system involving automated external defibrillators (AEDs), which are portable, life-saving devices designed to treat people experiencing sudden cardiac arrest. The goal is to achieve the world's highest rate of favorable neurological outcomes after sudden cardiac arrest.

The diagrams in the upper row show the current situation. If an individual finds a person who has collapsed, the only people who can help are those who happen to be passing by. If they are unfamiliar with cardiopulmonary resuscitation (CPR), they can only watch until an ambulance arrives at the scene. In cases of cardiac arrest due to ventricular fibrillation (VF), without first aid chest compressions by those present at the scene, the rhythm will change to a non-shockable rhythm that is not amenable to AED therapy by the time the ambulance arrives.

As shown in the bottom row panel, if a person who witnesses a cardiac arrest presses the SOS button, the location of the SOS button and the location of the nearest AED will be shown on the smartphone application of a pre-registered first responder who has received CPR training. This nearby first responder will quickly go to the scene with the AED and perform $\mathrm{CPR}$. If the cardiac arrest is due to VF, the AED will be effective. It is expected that the patient will achieve return of spontaneous circulation (ROSC) by the time the emergency team arrives.

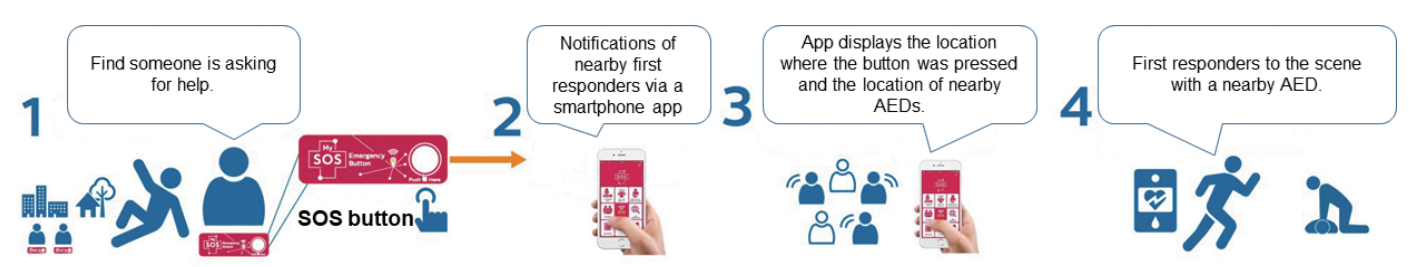

Fig. 2 Process after the SOS button has been pressed.

1. When you find a person who needs help, press the SOS button.

2. The My SOS application on the registered first smartphone will be automatically notified simultaneously.

3. The My SOS application displays the location of the SOS button activation and the location of nearby AEDs.

4. The first responders go to the scene with the nearby AED.

AED: automated external defibrillator.

an ambulance to be dispatched to the scene from the fire control center, the system notifies the smartphone of a first responder who has registered in advance and requests that an ambulance be dispatched to the scene with an AED transported from the fire control center ${ }^{8-14)}$. The difference between this system and the SOS button system is that the former uses the command and control center of the fire station (Fig. 5). By contrast, the latter is the world's first attempt at a system that sends messages directly to numerous or several first responders when the SOS button is pressed, without needing to go through the fire command center. 


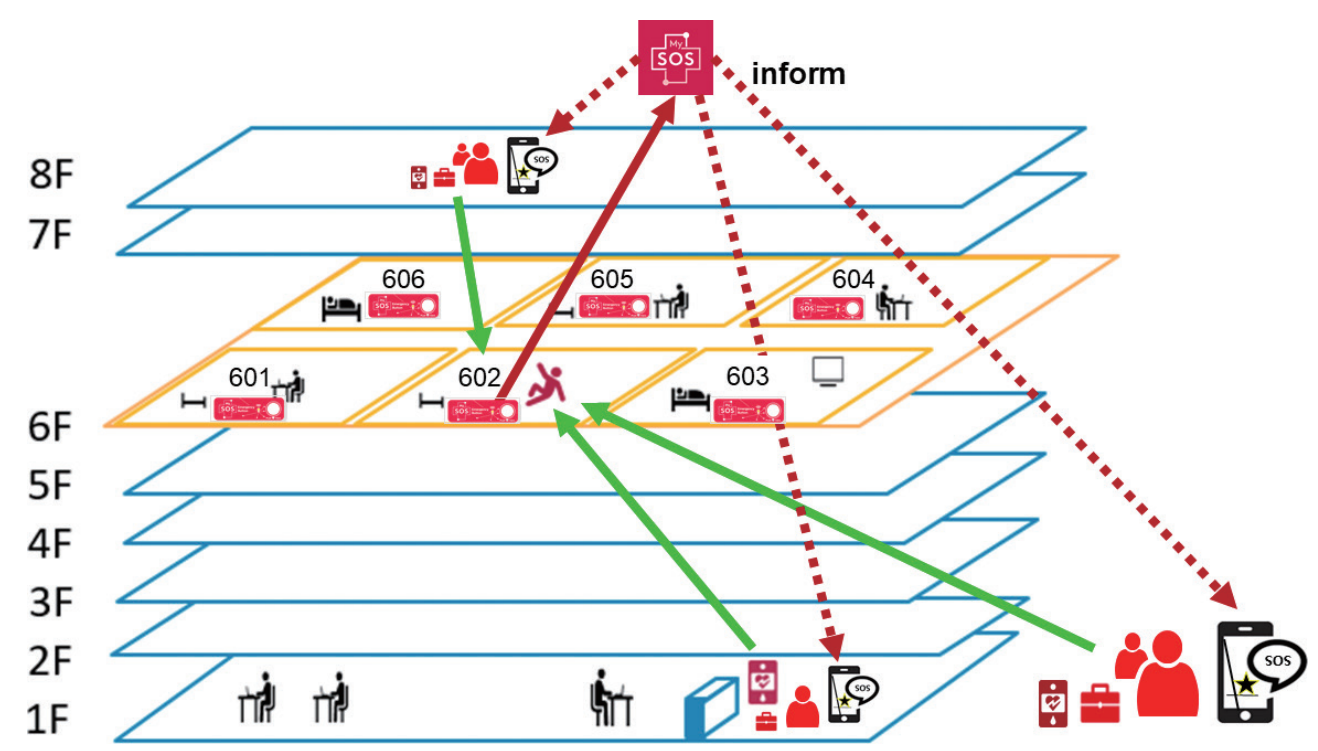

Fig. 3 Non-GPS type (space-axis support system).

The current SOS button system can be used two-dimensional GPS, it is unable to determine the space-axis location. The My SOS application notifies first responders of the patient's location, including the room number.

The red arrow indicates the activation of an SOS button in room 602 .

The dotted red arrows indicate the flow of information to each first responder; this information includes the floor number and room name, which indicate the location of SOS activation.

When responders receive the information from the My SOS application, they take a nearby AED and rush to the relevant room (green arrows).

GPS: Global Positioning System

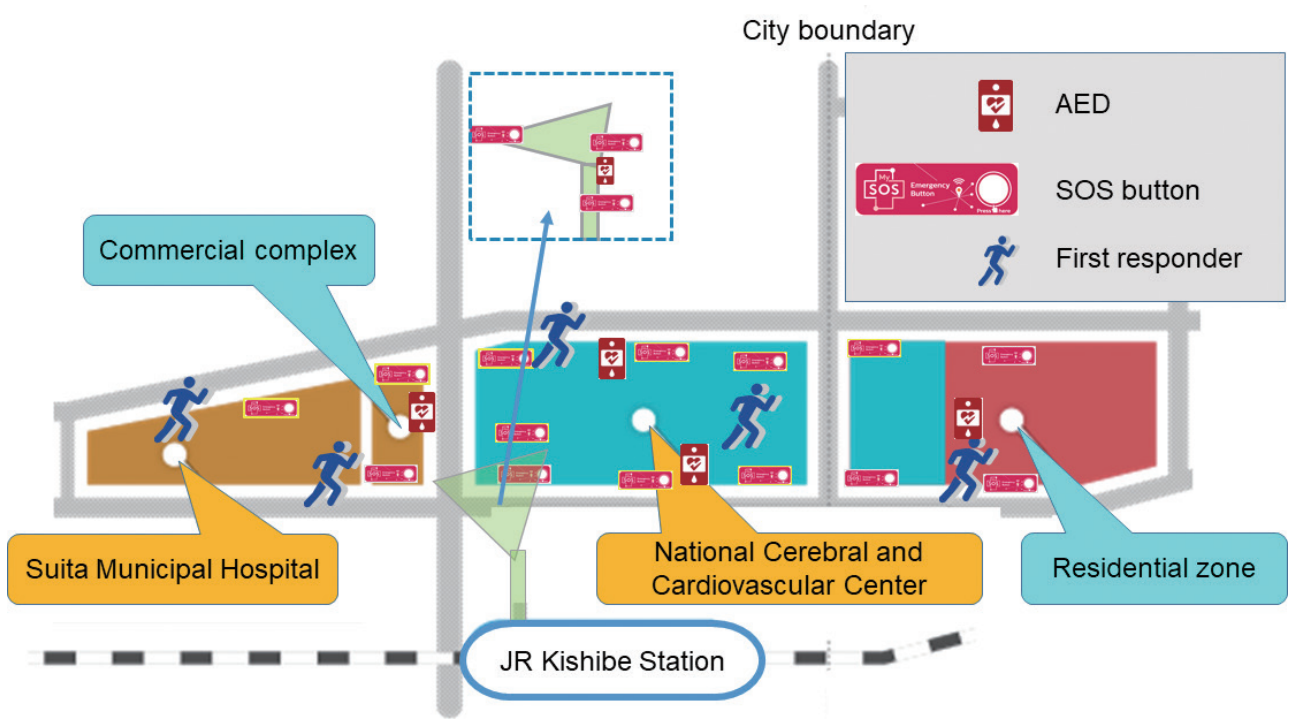

Fig. 4 Locations of AEDs and SOS buttons.

Kento is a world-class medical cluster consisting of an urban residential zone, a commercial complex, Suita Municipal Hospital, and the National Cerebral and Cardiovascular Center at its core. It is situated on 30 hectares of land in front of the JR Kishibe Station. Five AEDs and 16 SOS buttons were installed in Kento on March 2, 2020. First responders are employees of nearby facilities and members of the public in the Kento area who have taken cardiopulmonary resuscitation training beforehand and understand the SOS button system and are willing to participate. 


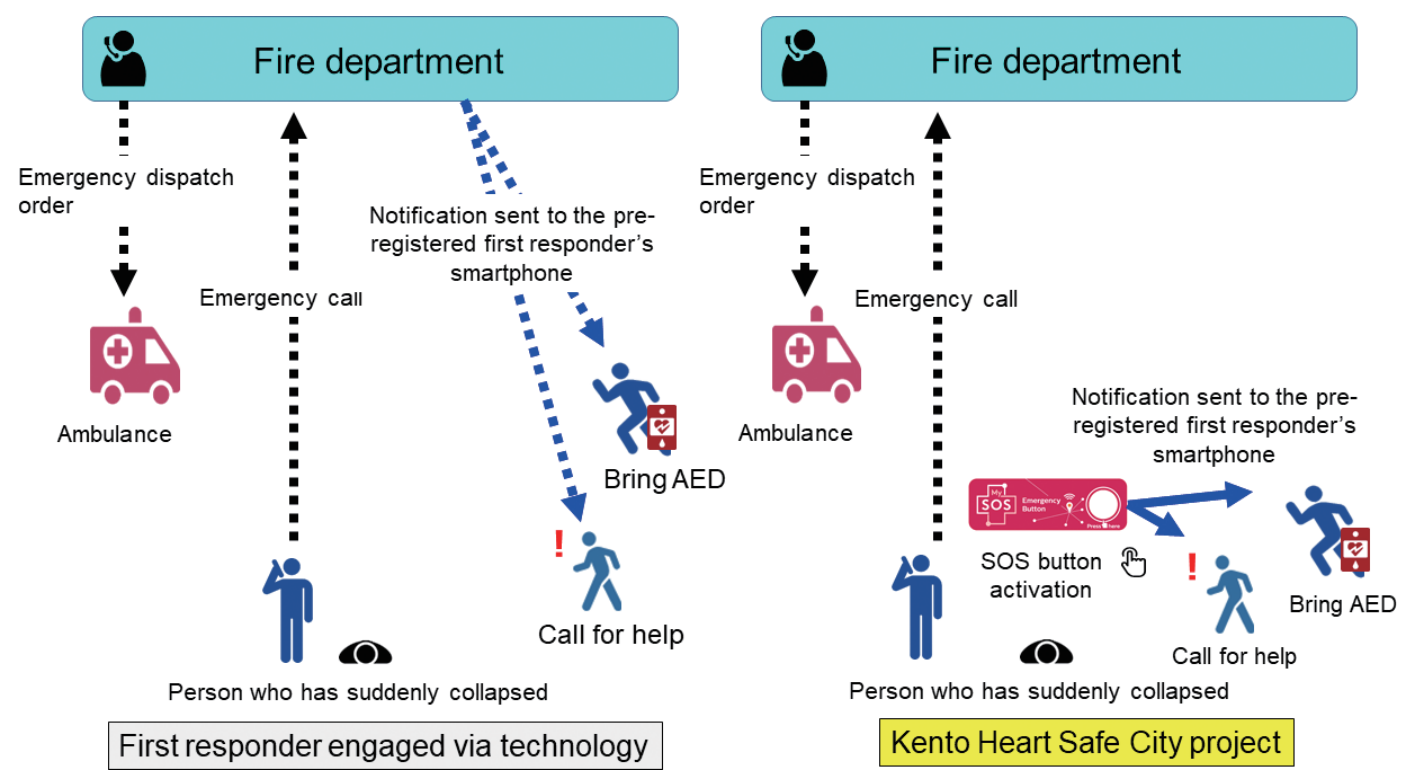

Fig. 5 Different methods of notifying first responders.

The latest cardiopulmonary resuscitation guidelines strongly recommend using smartphone technology to cooperate with pre-registered citizens in the vicinity of a suspected out-of-hospital cardiac arrest (left). The Kento Heart Safe City project is expected to deliver emergency medical support and AEDs to the scene more quickly because it takes a shorter time for first responders to be directly notified after the SOS button has been activation, without relaying the information via the fire control center (right).

AED: automated external defibrillator.

\section{Ongoing study design}

To verify whether first responders are able to respond to the aforementioned first-aid system that uses a newly developed emergency SOS button (Fig. 2), we conducted a single-center, prospective, observational study. Participants who experience a bystander-witnessed out-of-hospital cardiac arrest (OHCA) of cardiac origin with a shockable heart rhythm will be resuscitated by a bystander or will require critical care in the Kento area (Fig. 4). If a cardiac arrest or an event that requires critical care is witnessed by a person who then presses the SOS button, the location of the SOS button and the location of the nearest AED will be shown on the smartphone application of a nearby pre-registered first responder who has received CPR training. This individual will quickly take the AED to the scene and perform CPR The primary outcomes are the first responder may or may not have arrive to the scene, and the duration from time the SOS button is pressed to the arrival of the first responder on the scene. The secondary outcome is a favorable neurological outcome in patients at 3 months after OHCA. A favorable neurological outcome is defined as a Cerebral Performance Category score of 1 (good cerebral performance) or 2 (moderate cerebral disability ${ }^{15,16)}$. The goal is to reduce the average time from the emergency call (SOS button activation) to the arrival of the emergency team at the scene, and achieve the highest rate of favorable neurological outcomes after sudden cardiac arrest.

\section{Conclusion}

The SOS button and smartphones in the Kento Heart Safe City project represent the first attempt in the world to utilize IoT. This system is expected to be used in the future, including at the Osaka-Kansai Expo in 2025.

\section{Acknowledgments}

The research is funded by the Cardiovascular Development Fund (Dr. Kunihiro Nishimura, Department of Epidemiology and Informatics, Division of Preventive Medicine [30-1-14 Subject: Research on the construction of a rapid learning health system through the visualization of cardiovascular disease information using natural language processing and AI technology]. The project is also funded by a grant from the Japan Society of Cardiology.

\section{Discloser statement}

Dr. Kunihiro Nishimura reports receiving grants from Philips Japan. The other authors have no financial interests to disclose and no conflicts of interest to declare.

\section{References}

1) Current Status of Emergency and Rescue I. Emergency: https:// 
www.fdma.go.jp/publication/rescue/items/kkkg_r01_01_kyukyu.pdf

2) SOS-KANTO Committee: Incidence of ventricular fibrillation in patients with out-of-hospital cardiac arrest in Japan : survey of survivors after out-of-hospital cardiac arrest in Kanto area (SOS-KANTO). Circ J 2005; 69: 1157-1162

3) Kitamura T, Kiyohara K, Sakai T, et al: Public-access defibrillation and out-of-hospital cardiac arrest in Japan. N Engl J Med 2016; 375: 1649-1659

4) Greif R, Bhanji F, Bigham BL, et al: Education, implementation, and teams: 2020 international consensus on cardiopulmonary resuscitation and emergency cardiovascular care science with treatment recommendations. Circulation 2020; 142: S222-S283

5) Kern KB, Colberg TP, Wunder CJ, et al: A local neighborhood volunteer network improves response times for simulated cardiac arrest Resuscitation 2019; 144: 131-136

6) Nakashima K, Noguchi T, Tahara Y, et al: Public-access defibrillation and neurological outcomes in patients with out-of-hospital cardiac arrest in Japan: a population-based cohort study. Lancet 2019; 394: 2255-2262

7) Kobayashi D, Sado J, Kiyohara K, et al: Public location and survival from out-of-hospital cardiac arrest in the public-access defibrillation era in Japan. J Cardiol 2020; 75: 97-104

8) Berglund E, Claesson A, Nordberg P, et al: A smartphone application for dispatch of lay responders to out-of-hospital cardiac arrests. Resuscitation 2018; 126: 160-165

9) Caputo ML, Muschietti S, Burkart R, et al: Lay persons alerted by mobile application system initiate earlier cardio-pulmonary resuscitation: a comparison with SMS-based system notification. Resuscitation 2017; 114: 73-78

10) Lee SY, Shin SD, Lee YJ, et al: Text message alert system and resuscitation outcomes after out-of-hospital cardiac arrest: a before-and-after population-based study. Resuscitation 2019; 138: 198-207

11) Pijls RW, Nelemans PJ, Rahel BM, et al: Factors modifying performance of a novel citizen text message alert system in improving sur- vival of out-of-hospital cardiac arrest. Eur Heart J Acute Cardiovasc Care 2018; 7: 397-404

12) Ringh M, Rosenqvist M, Hollenberg J, et al: Mobile-phone dispatch of laypersons for CPR in out-of-hospital cardiac arrest. N Engl J Med 2015; 372: 2316-2325

13) Stroop R, Kerner T, Strickmann B, et al: Mobile phone-based alerting of CPR-trained volunteers simultaneously with the ambulance can reduce the resuscitation-free interval and improve outcome after out-of-hospital cardiac arrest: a German, population-based cohort study. Resuscitation 2020; 147: 57-64

14) Zijlstra JA, Stieglis R, Riedijk F, et al: Local lay rescuers with AEDs, alerted by text messages, contribute to early defibrillation in a Dutch out-of-hospital cardiac arrest dispatch system. Resuscitation 2014; 85: $1444-1449$

15) Jacobs I, Nadkarni V, Bahr J, et al: Cardiac arrest and cardiopulmonary resuscitation outcome reports: update and simplification of the Utstein templates for resuscitation registries: a statement for healthcare professionals from a task force of the International Liaison Committee on Resuscitation (American Heart Association, European Resuscitation Council, Australian Resuscitation Council, New Zealand Resuscitation Council, Heart and Stroke Foundation of Canada, InterAmerican Heart Foundation, Resuscitation Councils of Southern Africa). Circulation 2004; 110: 3385-3397

16) Perkins GD, Jacobs IG, Nadkarni V, et al: Cardiac arrest and cardiopulmonary resuscitation outcome reports: update of the Utstein Resuscitation Registry Templates for Out-of-Hospital Cardiac Arrest: a statement for healthcare professionals from a task force of the International Liaison Committee on Resuscitation (American Heart Association, European Resuscitation Council, Australian and New Zealand Council on Resuscitation, Heart and Stroke Foundation of Canada, InterAmerican Heart Foundation, Resuscitation Council of Southern Africa, Resuscitation Council of Asia); and the American Heart Association Emergency Cardiovascular Care Committee and the Council on Cardiopulmonary, Critical Care, Perioperative and Resuscitation. Circulation 2015; 132: 1286-1300 